\title{
Die Politik sozialkonstruktivistischer Normenforschung und ihre poststrukturalistische Alternative
}

\author{
Judith Renner
}

\section{Einleitung}

Normen scheinen auf den ersten Blick etwas Gutes zu sein. Sie beschreiben Verhaltensweisen, die wir als normal oder auch als angemessen betrachten. Meist haben wir diese Normen von klein auf kennengelernt und fügen uns ihnen deshalb in vielen Fällen freiwillig. Ähnliche Grundgedanken scheinen auch dem Mainstream ${ }^{1}$ der sozialkonstruktivistischen Normenforschung zugrunde zu liegen, denn auch er geht relativ unkritisch und affirmativ an diejenigen Normen heran, die er untersucht, und behandelt diese oft als gegebene Verhaltensstandards, deren Geltungsansprüche und Konsequenzen weitgehend unhinterfragt bleiben (vgl. etwa Klotz 1995; Finnemore/Sikkink 1998; Keck/Sikkink 1998; Risse, et al. 1999; Park 2005). Die zentralen Forschungsfragen, die sich ein großer Teil der sozialkonstruktivistischen Normenforschung stellt, drehen sich vor allem darum, wie scheinbar existente Normen sich global verbreiten können (zur Adaption globaler Normen auf lokaler Ebene siehe auch den Beitrag von Lisbeth Zimmermann in diesem Band) und welche Strategien sogenannte NormunternehmerInnen anwenden, um neue Staaten von als gut und gültig empfundenen Normen zu überzeugen.

Die affirmative Haltung der konstruktivistischen Normenforschung zieht Konsequenzen nach sich, da sie, indem sie die untersuchten Normen als gegeben nimmt, diese implizit als (gültige) Normen bestätigt und naturalisiert. Wird eine geteilte Bedeutung in der Forschung als Norm anerkannt und als solche untersucht, so wird ihr normativer und wünschenswerter Charakter kaum hinterfragt. Kritische Fragen hinsichtlich dieser Norm rücken tendenziell in den Hintergrund. Dazu gehören etwa Fragen nach der Plausibilität oder der Wünschbarkeit bestimmter Normen, ge-

1 Unter Mainstream der Normenforschung verstehe ich in diesem Beitrag vor allem diejenigen Beiträge, die sich, v.a. aus gemäßigt konstruktivistischer Perspektive, darauf fokussieren, die Diffusion und Wirkung von Normen zu erklären. 
nauso wie Fragen nach möglichen negativen und unerwünschten Konsequenzen und der Macht, die von ihnen ausgeübt wird.

Kurz gesagt argumentiere ich im vorliegenden Beitrag, dass Normenforschung als politische Praxis zu verstehen ist. Sie wirkt - zumeist unreflektiert - an der Rekonstruktion und Stärkung bestimmter normativer Gefüge mit und vernachlässigt deren Kontingenz und vor allem deren (macht)politische Aspekte.

Dieser Beitrag zielt darauf ab, eine Alternative zur affirmativen Haltung konstruktivistischer Normenforschung zu entwickeln, indem er eine theoretische Perspektive vorschlägt, die einen dezidiert kritischen Blick auf globale hegemoniale Normen ermöglicht. Ein solch kritischer Blick ist meines Erachtens eine wichtige Ergänzung zur konstruktivistischen Normenforschung, da wir dazu tendieren, insbesondere diejenigen Dinge, die wir im Alltag und im Laufe unserer Sozialisierung als gut und richtig oder auch als normal kennenlernen, kaum noch zu hinterfragen, sondern unreflektiert zu akzeptieren und zu praktizieren. Die hier vorgeschlagene kritische Perspektive auf Normen im Allgemeinen und die globale Versöhnungsnorm im Besonderen soll dabei helfen, die akzeptierte Autorität und Gültigkeit dieser Normen zu hinterfragen und die Möglichkeit eröffnen, alternative Wege politischen Handelns denkbar zu machen (zur Umstrittenheit von Normen siehe auch die Beiträge von Gholiagha, Hansen-Magnusson und Hofius sowie Schillinger und Holger Niemann in diesem Band).

Als theoretische Grundlage für ein solches kritisches Projekt, so argumentiere ich, bietet sich die poststrukturalistische Diskurstheorie an, die als radikale und politische Form konstruktivistischen Denkens verstanden werden kann. Eine poststrukturalistische Perspektive geht davon aus, dass soziale Realität nicht gegeben, sondern immer sozial konstruiert ist. Somit kann auch Wissenschaft niemals objektiv und neutral sein (vgl. Cox 1981). Der politische Charakter sozialwissenschaftlicher Forschung ist unvermeidbar, da jegliche Forschung als politische Praxis verstanden werden muss, die durch ihre theoretischen Annahmen und ihre Forschungspraktiken bestimmte Aspekte sozialer Realität als gegeben annimmt und reproduziert, während sie andere zugleich hinterfragt. Was ich im Folgenden vorschlage ist daher kein Versuch, ein neutrales Programm zur Erforschung globaler Normen zu entwickeln. Stattdessen möchte ich eine Möglichkeit schaffen, scheinbar akzeptierte Normen kritisch zu beleuchten, sie zu denaturalisieren, ihre Geltungsansprüche zu hinterfragen und die Konsequenzen aufzudecken, die sowohl in der Wissenschaft als auch in der politischen Praxis oft übersehen werden.

Im ersten Teil dieses Beitrags setze ich mich kritisch mit der konstruktivistischen Normenforschung auseinander. Ich argumentiere, dass diese 
Forschung insofern politisch problematisch ist, da sie - wenn auch vielleicht unbewusst und unwillentlich - die empirischen Normen, die sie untersucht, in ihrer Existenz als Norm als gegeben nimmt und sie damit implizit naturalisiert und bestätigt. Dieser Kritikpunkt ist dabei bereits aus einer poststrukturalistischen Perspektive formuliert, die ich im zweiten Teil des Beitrags als fruchtbare und vor allem kritische Alternative für die Normenforschung vorschlage. Poststrukturalistische Ansätze ermöglichen es, die Autorität und die Geltungsansprüche weitgehend akzeptierter Normen zu hinterfragen und ihre historische Kontingenz wie auch ihre diskursive Macht zu beleuchten. Sie konzeptualisieren globale Normen - wie alle anderen Aspekte sozialer Realität - als Bestandteile hegemonialer Diskurse, die bestimmte Handlungen als gut, legitim oder normal erscheinen lassen, während sie alternative Handlungsweisen de-legitimieren oder marginalisieren. Diese produktiven und repressiven Aspekte diskursiver Macht aufzudecken ist ein Kernanliegen der hier vorgeschlagenen poststrukturalistischen Normenforschung. ${ }^{2}$

Um die Vorgehensweise und die Möglichkeiten einer solchen poststrukturalistischen Normenforschung zu illustrieren, skizziere ich schließlich im dritten Teil des Beitrags die Herausbildung und Wirkung einer globalen Versöhnungsnorm. Diese ist vor allem im Kontext von Transitional Justice und Postconflict Peacebuilding hegemonial geworden und legt nahe, dass das Streben nach nationaler Versöhnung und die Schaffung von Wahrheits- und Versöhnungskommissionen ein gutes und angemessenes Verhalten nach Krieg und Unterdrückung sei. Aus poststrukturalistischer Perspektive lässt sich dies jedoch in Zweifel ziehen. Vielmehr argumentiere ich, dass die globale Versöhnungsnorm Teil eines historisch gewachsenen Diskurses ist, der das kontingente Resultat politischer Auseinandersetzungen und Artikulationsakte ist und der weder einen gegebenen Geltungsanspruch noch einen inhärenten moralischen Wert besitzt. Ein Blick auf die produktive und repressive Macht dieses Diskurses zeigt zudem, wie die Versöhnungspraxis weitgehend übersehene und möglicherweise unerwünschte Konsequenzen zeitigt, indem sie tendenziell de-politisierend auf Postkonfliktgesellschaften wirkt. Sie überschreibt die politischen Identitäten der Menschen in Postkonfliktgesellschaften und bringt damit politi-

2 Diese beiden Kernanliegen können gewinnbringend erweitert werden, indem man etwa auch Fragen nach Dominanz, Antagonismus oder auch Inklusion und Exklusion im Artikulationsprozess integriert. Der Umfang des vorliegenden Beitrags lässt dies leider nicht zu. Ich danke Antonia Graf für diese Hinweise. 
sche Forderungen zum Schweigen, die von diesen Menschen erhoben werden.

\section{Ein poststrukturalistischer Blick auf Normenforschung}

Normen sind seit langem zentraler Untersuchungsgegenstand vieler konstruktivistischer ForscherInnen, und insbesondere die Frage nach der Verbreitung neuer Normen hat seit geraumer Zeit an Popularität gewonnen. Mittlerweile hat sich ein umfassender Literaturkanon entwickelt, der die Herausbildung sozialer Normen und die Bedingungen ihrer globalen Diffusion untersucht. ${ }^{3}$

Die Kritik dieses Beitrags fokussiert sich auf zwei prominente Erklärungsmodelle der Diffusionsforschung, die normativen Wandel zum großen Teil auf die Aktivitäten verschiedener transnationaler AkteurInnen, etwa NormunternehmerInnen, Advocacy Networks oder epistemische Gemeinschaften, zurückführen. ${ }^{4}$ Das erste Modell nimmt an, dass Normen durch strategisches Handeln von NormunternehmerInnen verbreitet werden, deren festes Ziel es ist, bestimmte Normen bei neuen AkteurInnen, primär Staaten, zu etablieren. Neue Normen sind demnach »actively built by agents having strong notions about appropriate or desirable behaviour in their community (Finnemore/Sikkink 1998: 896). Dafür, so die Annahme, greifen NormunternehmerInnen rational auf verschiedene politische Strategien zurück, etwa diskursives Framing, Druckausübung oder auch das Anprangern von normverletzenden Staaten (Risse/Sikkink 1999; Finnemore/Sikkink 1998; Keck/Sikkink 1998).

Das zweite Erklärungsmodell schlägt dagegen vor, dass nicht strategisches, sondern verständigungsorientiertes Handeln von NormunternehmerInnen und echte Überzeugungsprozesse der Schlüssel zum Verständnis normativen Wandels seien (vgl. etwa (Deitelhoff 2009; 2006; Payne 2001). Die hier verwendeten Überzeugungs- und Argumentationskonzepte bauen häufig auf einem Habermas'schen Modell sozialer Interaktion und Delibe-

3 Vgl. z. B. Finnemore/Sikkink 1998; Deitelhoff 2006; Price 1995; Florini 1996; Sandholtz 2008; Bailey 2008 .

4 Einige konstruktivistische ForscherInnen haben in den letzten Jahren allerdings auch alternative Ansätze entwickelt, die normativen Wandel vielmehr als kontingentes Produkt der Kämpfe und Auseinandersetzungen betrachten, die kontinuierlich um die Interpretation kollektiver Bedeutungen stattfinden und sich in jeglicher Art sozialer Interaktion manifestieren; siehe z.B. Sandholtz 2008; Wiener 2004; 2007a; 2007b. 
ration auf und nehmen an, dass AkteurInnen nicht strategisch handeln, also auf der Basis stabiler Interessen, sondern vielmehr gemäß einer argumentativen Rationalität (Risse 2000: 2-14; Deitelhoff 2009: 35). Überzeugung, so wird angenommen, findet hier durch einen wahren Gesinnungswandel statt, der ohne Druck oder Zwang vonstattengeht. Die Normen, die in diesen Überzeugungsprozessen und rationalen Diskursen entstehen, seien dann die Produkte der " noncoercive coercion" of the better argument" (Deitelhoff 2009: 43). Ob strategisches Framing oder wahre Überzeugung, an beiden Modellen lässt sich kritisieren, dass diese - wenn auch auf unterschiedliche Art und Weise - durch ihre Erklärungen eine weitgehend unreflektierte Politik betreiben, indem sie die Wünschbarkeit und normative Geltung von Normen unhinterfragt lassen oder diese aktiv legitimieren. Konstruktivistische NormenforscherInnen werden somit zu KomplizInnen einer ganz bestimmten Art von Realitätspolitik, die die untersuchten Normen, genauso wie die soziale Realität, die sie naturalisieren, reproduziert und stärkt.

Das erste, strategisch argumentierende Modell normativen Wandels nimmt die untersuchten Normen als gegeben und lässt die Frage offen, wie deren normative Bedeutungen konstruiert wurden, so dass zielgerichtetes, strategisches Handeln in ihrem Namen erst möglich wird. Dies bringt nicht nur eine unnötige analytische Einschränkung mit sich, da es der/dem Forschenden den Blick auf die historisch kontingente Natur und die konstitutive Wirkung normativer Bedeutungen verstellt (Price 1995: 87). Vielmehr verdeckt die Vernachlässigung der historisch kontingenten Entstehung von Normen auch deren inhärent politischen Charakter: Normen können als produktive Diskurse oder, besser, als Bestandteile derselben verstanden werden, die bestimmte Praktiken und Identitäten naturalisieren und legitimieren, während sie zugleich andere delegitimieren. Eine Untersuchung, die speziell auf den Konstruktionsprozess von Normen abzielt, würde es insofern ermöglichen, die historische Produktivität sozialer Normen zu erforschen und die versteckteren konstitutiven Effekte aufzudecken. Sie kann, in den Worten von Jenny Edkins, dabei helfen, die "politischen Technologien" (Edkins 1999: 9) zu entlarven, die durch Normen produziert und naturalisiert werden und heute gerne übersehen werden, da die Norm genauso wie die soziale Realität, die sie produziert, seit langem als gültige soziale Bedeutungen akzeptiert und als gegeben angenommen werden. Indem das strategische Erklärungsmodell annimmt, dass bestimmte Normen einfach da sind und strategisch von NormunternehmerInnen propagiert werden können, naturalisiert es diese Normen gewissermaßen in ihrer Existenz als Norm an sich, inklusive ihrer Geltungsansprüche. Dieses Prozedere hat insofern politische Implikationen, als es die nor- 
mative Qualität einer historisch kontingenten Norm und damit auch die soziale Realität bestätigt, die durch sie produziert wird.

Ein noch stärkerer politischer Effekt kann hinsichtlich der Überzeugungsansätze beobachtet werden, da diese die untersuchte Norm nicht nur naturalisieren, sondern auch implizit legitimieren. Indem Überzeugungsansätze auf dem Habermas'schen Argumentationsmodell und dem Konzept des rationalen Diskurses aufbauen, um die Herausbildung und Institutionalisierung einer spezifischen Norm zu erklären, nutzen sie ein eigentlich normativ-kritisches Instrument, das versucht, ein ethisches Fundament für ein realweltlich begründbares Moralsystem anzubieten (Hanrieder 2008: 168). Moralisch begründbare und akzeptable Normen können demnach durch bestimmte prozedurale Bedingungen erreicht werden, nämlich Argumentation in einem rationalen Diskurs, der jeder/m einzelnen TeilnehmerIn den gleichen Zugang, die gleichen Machtpotenziale und Schutz vor Diskriminierung garantiert und Konsens durch die Überzeugungskraft des besten Arguments herbeiführt.

Die Übertragung dieses normativen Modells und seine Verwendung für die Erklärung empirischer Normentstehungsprozesse moralisiert diese Prozesse jedoch und legitimiert empirisch akzeptierte Normen ex post (Hanrieder 2008): Wenn empirische Prozesse der Normentstehung im Sinne von rationalen und gerechten Diskursen interpretiert werden, so werden de facto hervorgebrachte Argumente zu guten oder gar besten Argumenten und empirisch entstehende Normen werden zu moralisch akzeptablen und scheinbar gerechten Normen. Hier wird die untersuchte Norm also nicht nur naturalisiert, sondern implizit für legitim erklärt, da sie scheinbar im Rahmen eines rationalen Diskurses entstanden ist. Kritische Fragen hinsichtlich der Wünschbarkeit einer Norm oder auch hinsichtlich ihrer Macht und eventueller negativer Konsequenzen werden dadurch erschwert und zunächst in den Hintergrund gerückt.

Die normative Prädisposition konstruktivistischer Normenforschung mag nicht beabsichtigt sein. Dennoch betreibt diese Forschung eine ganz bestimmte Realitätspolitik, indem sie diejenigen Bedeutungen, die sie untersucht, naturalisiert und stärkt, und dadurch auch die größeren Wertbestände legitimiert und reproduziert, in die diese Bedeutungen eingebunden sind. Konstruktivistische Normenforschung bietet damit keinen neutralen, objektiven Blick auf die Welt, die sie untersucht; stattdessen ist sie an der Konstruktion von Wirklichkeit beteiligt und trägt zur Reproduktion und Normalisierung hegemonialer Wertbestände und Machtstrukturen bei (vgl. Engelkamp, et al. 2012). 


\section{Poststrukturalismus als Alternative: Ein kritischer Blick auf globale Normen}

Die obige Kritik am zumeist unreflektierten politischen Charakter konstruktivistischer Normenforschung ist aus einer poststrukturalistischen Perspektive formuliert, die ich im Folgenden als alternative und kritische Herangehensweise für die Erforschung globaler Normen vorschlage. Die poststrukturalistische Diskurstheorie bietet sich insofern als Grundlage für ein alternatives Forschungsprogramm zur affirmativen Haltung konstruktivistischer Normenforschung an, als sie einen dezidiert kritischen Blick auf hegemoniale Normen ermöglicht.

Aus poststrukturalistischer Sicht repräsentieren Normen - genauso wie alle anderen sozialen Bedeutungen - Komponenten historisch kontingenter Diskurse, die durch soziale Praktiken, insbesondere Sprache, produziert und reproduziert werden. Was AkteurInnen als richtig, angemessen oder auch normal verstehen, wird von hegemonialen Diskursen vorgegeben, die bestimmte Praktiken konstituieren und naturalisieren und sie gut oder angemessen erscheinen lassen. Hegemoniale Diskurse sind aber keine neutrale Abbildung von Wirklichkeit, sondern werden in der poststrukturalistischen Forschung als hoch politisch betrachtet, da ihre Hegemonie nur auf Kosten anderer Versionen sozialer Realität bestehen kann, die zugleich ausgeschlossen, marginalisiert und delegitimiert werden. Soziale Realität und damit auch soziale Normen bergen insofern immer eine repressive Seite, die aufzudecken zentrales Anliegen poststrukturalistischer Forschung ist. Grundsätzlich legt eine poststrukturalistisch inspirierte Normenforschung ihren Fokus also nicht nur auf die Normen, die kritisch beleuchtet werden sollen, sondern auf die Diskurse, die diesen Normen zugrunde liegen und auf deren produktive und repressive Macht, d.h. ihre Fähigkeit, einerseits eine bestimmte Konstruktion sozialer Realität als gut oder auch wahr erscheinen zu lassen und andererseits alternative Werthaltungen und Interpretationen von Realität zu marginalisieren und zu delegitimieren (siehe genauer weiter unten). Zentrales Anliegen bei der Anwendung einer solchen Perspektive ist es, zu rekonstruieren, wie diese Diskurse entstehen und hegemonial werden, wie sie soziale Realität, z.B. Normen, produzieren und schließlich, welche Konsequenzen diese Konstruktionen für soziale Realität bzw. für die Weltpolitik mit sich bringen. Wie ein poststrukturalistisches Forschungsdesign für die kritische Untersuchung globaler Normen aussehen kann, skizziere ich auf den folgenden Seiten. 


\subsection{Poststrukturalistische Grundannabmen}

Die poststrukturalistische Diskurstheorie kann als radikale und politische Form konstruktivistischen Denkens verstanden werden, die auf einer antiessentialistischen Ontologie und einer anti-fundationalen Epistemologie (Torfing 2005: 13) aufbaut. Ontologisch verwirft sie die Annahme, dass soziale Realität auf eine stabile und gegebene Essenz zurückgeführt werden kann und geht stattdessen davon aus, dass Realität immer eine soziale Konstruktion ist, die durch Diskurse produziert und stabilisiert wird. Epistemologisch nimmt sie an, dass es keine gegebene und fixe Wahrheit über die Welt geben kann. Wabrheit ist immer nur im Kontext von und bedingt durch hegemoniale Diskurse möglich, die bestimmen, was als wahr oder falsch gelten kann (Torfing 2005: 14). In diesem Sinne muss auch Wissenschaft immer als politisches Projekt begriffen werden, das mit seinen Wabrheiten und Erkenntnissen auf bestimmten hegemonialen Diskursen aufbaut und diese stärkt und reproduziert.

Aus poststrukturalistischer Sicht ist Diskurs die zentrale konstitutive Kategorie sozialer Realität, da es nur in Diskursen und durch Diskurse möglich ist, Bedeutungen temporär zu stabilisieren und die materielle Umwelt bedeutungsvoll werden zu lassen (Epstein 2008: 2; Howarth/Stavrakakis 2000; Torfing 2005). Diskurse können nach Ernesto Laclau und Chantal Mouffe als relativ stabile Bedeutungsarrangements verstanden werden, als »structured totalit[ies]«, in denen Bedeutungen und soziale Identitäten in relationalen Bedeutungssystemen angeordnet werden. Laclau und Mouffe zufolge werden Diskurse in und durch Knotenpunkte stabilisiert, die durch sogenannte leere Signifikanten, semantisch vage soziale Ideale wie etwa Demokratie, Gerechtigkeit, oder - wie ich im Folgenden argumentiere Versöbnung sprachlich repräsentiert werden. Solche leeren Signifikanten stabilisieren einen Diskurs, indem sie als Ankerpunkt für alle anderen Signifikanten des Diskurses fungieren und diese durch Äquivalenzbeziehungen an sich binden. Insgesamt werden Diskurse durch zwei gegenläufige sprachliche Logiken zusammengehalten: Die Logik der Äquivalenz, die den leeren Signifikanten mit den anderen Signifikanten des Diskurses in eine positive semantische Beziehung setzt, und die Logik der Differenz, die diese Signifikanten wiederum voneinander unterscheidet und die Spannung des diskursiven Systems aufrecht erhält (Laclau/Mouffe 2001: 127-134). Diese Logiken sind nicht nur für die Bedeutungsbildung, sondern auch für die Ab- und Ausgrenzungen eines Diskurses verantwortlich: was radikal vom leeren Signifikanten unterschieden und damit von seiner Äquivalenzkette abgegrenzt wird, wird als das oftmals illegitime Andere konstruiert und an die diskursiven Grenzen verbannt (Laclau 2007: 38). 
Diskurse werden durch Artikulationen produziert und reproduziert, d.h. durch »any practice establishing a relation among elements such that their identity is modified as a result of the articulatory practice (Laclau/ Mouffe 2001: 105). Nach Laclau und Mouffe enthält jede soziale Praxis, linguistisch wie nicht-linguistisch, eine artikulatorische Dimension, da jede soziale Handlung bestimmte Bedeutungen und Beziehungen zwischen Bedeutungen bestätigt und reproduziert, während andere zugleich hinterfragt und modifiziert werden (Laclau/Mouffe 2001: 113). Die AutorInnen unterscheiden zudem eine besondere Form von Artikulation, die sie hegemoniale Kämpfe nennen, und die insbesondere in Situationen sozialer Krise und extremer Umstrittenheit zur Artikulation neuer Diskurse führen. Solche hegemonialen Kämpfe werden durch politische Auseinandersetzungen ausgetragen, in deren Verlauf ein Signifikant zum neuen, hegemonialen leeren Signifikanten erhoben und mit anderen Signifikanten in eine Äquivalenzbeziehung gesetzt wird. Damit verbunden ist die Privilegierung dieses Signifikanten über konkurrierende Ideale und zugleich deren Ausgrenzung als illegitimes Anderes (Laclau/Mouffe 2001: 135-142; Laclau 2004: 318-321; Howarth 2004: 258-261).

Soziale Realität, also das, was wir als objektiv wahr oder natürlich verstehen, wird durch hegemoniale Diskurse produziert, die insofern mächtig sind, als sie eine bestimmte, historisch kontingente Interpretation sozialer Realität fixieren und institutionalisieren, während sie zugleich andere Versionen sozialer Realität ausschließen, unterdrücken und marginalisieren (Howarth/Stavrakakis 2000: 4; Torfing 2005: 14-15). In anderen Worten: Hegemoniale Diskurse machen einen Unterschied, da die soziale Realität, die von ihnen konstruiert wird, genauso gut auch anders konstruiert werden könnte (Epstein 2008: 9). Die produktive Macht hegemonialer Diskurse liegt demnach in ihrer Fähigkeit, eine diskursiv produzierte, dabei aber völlig kontingente soziale Realität, bestehend aus Subjekten, Objekten und Praktiken objektiv wahr und gegeben erscheinen zulassen.

Zugleich wird es uns aber auch nur möglich, eben diese Subjektpositionen einzunehmen und eben jene Praktiken auszuüben, die vom hegemonialen Diskurs zur Verfügung gestellt werden, während andere Subjektpositionen oder Praktiken als unnatürlich, anormal oder schlecht ausgeschlossen und delegitimiert werden. Die Macht eines hegemonialen Diskurses birgt also immer auch eine repressive Seite, und die Subjekte, Objekte und Praktiken, die im Diskurs produziert werden, sind nicht unproblematisch, da sie alternative Konstruktionen unterdrücken und marginalisieren. Die Hegemonie eines Diskurses basiert damit auf einem permanenten Kampf dieses Diskurses gegen andere Konstruktionen sozialer Realität, die immer wieder unterdrückt und delegitimiert werden müssen, um die Hegemonie 
eines Diskurses zu erhalten. In anderen Worten, Diskurse und alle soziale Objektivität, die von ihnen produziert wird, sind inhärent politisch; ihre Formierung impliziert die Ausübung von Macht und der Prozess ihrer Sedimentierung ist die Institutionalisierung von Machtbeziehungen (Howarth/Stavrakakis 2000: 9).

Die Anerkennung der inhärenten Kontingenz, Macht und Politik hegemonialer Diskurse hat entscheidende Konsequenzen dafür, wie wir soziale Realität, internationale Politik und auch Normen verstehen und analysieren. Erstens impliziert sie, dass soziale Realität und das, was wir als Normen begreifen, niemals als neutral betrachtet werden kann, sondern immer als Produkt mächtiger, aber kontingenter Diskurse zu verstehen ist, deren Hegemonie auf dem Ausschluss alternativer Konstruktionen basiert. Diese anderen, unterdrückten und zugunsten des hegemonialen Diskurses delegitimierten Versionen sozialer Realität - etwa die ausgeschlossenen Verhaltensstandards, die es nicht zur Norm geschafft haben, oder die Subjektpositionen, die durch den hegemonialen Diskurs überschrieben wurden - gilt es in einer kritischen Studie aufzudecken, um die Kontingenz und die Politik des hegemonialen Diskurses zu enthüllen und alternative Konstruktionen von Realität und legitimer sozialer Ordnung aufzuzeigen, die ebenso gut möglich gewesen wären.

Zweitens ist das Konzept der diskursiven Macht zentral für die Zuweisung von Verantwortung (vgl. Guzzini 2000; 2005: 511). Indem wir als WissenschaftlerInnen einen Diskurs als mächtig beschreiben und zugleich annehmen, dass wir, als AkteurInnen im sozialen Alltag durch unsere Sprache und unsere Praktiken an der (Re-)Produktion dieses Diskurses beteiligt sind, implizieren wir, dass wir einen Teil der Verantwortung dafür tragen, wie unsere soziale Realität ist. Wenn wir z.B. weitgehend akzeptierte Normen unseres eigenen Kulturkreises als gegeben nehmen und naturalisieren, dann stärken wir zugleich den Diskurs, in den diese Normen eingebunden sind und reproduzieren sein Machtgefüge, welches z.B. westliche Normen privilegiert, während Normen und Praktiken aus anderen kulturellen Kontexten delegitimiert und als schlecht aufgefasst werden. Auf der anderen Seite sind wir, als ForscherInnen, durch eine dezidiert kritische Forschungspraxis wie die poststrukturalistische Hegemoniekritik in der Lage, bewusst den politischen Charakter scheinbar gültiger und neutraler Normen aufzudecken, ihre Wirkung zu enthüllen und sie und den hegemonialen Diskurs, in den sie eingebunden sind, dadurch zu re-politisieren, d.h. wieder umstritten zu machen. 


\subsection{Skizze einer poststrukturalistisch inspirierten Normenforschung}

Die poststrukturalistische Diskurstheorie bietet nun die Möglichkeit, das, was genuin als globale Normen bezeichnet wird, kritisch zu beleuchten, indem sie zwei zentrale Forschungsschritte vorschlägt: Erstens erlaubt sie es, den Geltungsanspruch scheinbar geteilter Normen zu hinterfragen, indem sie die Existenz von Normen und deren feststehenden Bedeutungen nicht als gegeben annimmt, sondern sie als Bestandteile historisch gewachsener, kontingenter Diskurse versteht, die das Resultat politischer und sozialer Auseinandersetzungen und Artikulationsprozesse sind und keinen inhärenten moralischen Wert besitzen. Die Kontingenz und Veränderbarkeit dieser Diskurse und damit die Kontingenz von Normen aufzuzeigen ist ein zentrales Anliegen der hier vorgeschlagenen poststrukturalistisch inspirierten Normenforschung.

Methodisch kann man dabei, wie von einigen kritischen ForscherInnen vorgeschlagen, etwa auf einen genealogischen Ansatz zurückgreifen, indem man die untersuchte Norm historisiert und diejenigen historischen Artikulationsprozesse rekonstruiert, durch die diese mit (wechselnden) Bedeutungen versehen und in den Stand eines autoritativen Wertes erhoben wurde (Milliken 1999; Price 1995). Wie Richard Price argumentiert, hilft ein genealogisches Vorgehen dabei, zu zeigen, dass

"what is most often found at the historical beginnings of things is not 'the moment of their greatest perfection, when they emerge dazzling from the hands of a creator'. Rather, that development of institutions often consists of rationally inexplicable events, 'fabricated in piecemeal fashion' out of the vicissitudes of history« (Price 1995: 86).

Aufbauend auf dem oben skizzierten, von Laclau und Mouffe inspirierten Diskursverständnis schlage ich als Methode zur Textanalyse die Analyse der semantischen Beziehungen vor, die zwischen Signifikanten artikuliert werden. Wie Norman Fairclough darlegt, eignet sich diese Methode dazu, Äquivalenz- und Differenzbeziehungen zwischen Signifikanten zu rekonstruieren und zu analysieren, welche Bedeutungseinheiten in eine Äquivalenzbeziehung mit dem leeren Signifkanten gesetzt werden und welche zu ihm in Kontrast gesetzt und damit ausgegrenzt werden (Fairclough 2003: 87-98). Dabei geht es eben nicht nur darum, die positive Bedeutungsproduktion nachzuvollziehen, d.h. den historischen Artikulationsprozess, durch den eine Norm konstruiert und damit geschaffen wird, sondern auch um die repressive Seite dieses Prozesses, d.h. um die anderen, alternativen Werte und Signifikanten, die durch die Privilegierung eines Signifi- 
kanten unterdrückt und delegitimiert werden, indem sie vom Diskurs ausgeschlossen werden.

Zweitens ermöglicht eine poststrukturalistisch inspirierte Perspektive, den politischen Charakter globaler Normen zu untersuchen und aufzudecken, welche produktive und repressive Macht von ihnen ausgeübt wird. Als Bestandteile hegemonialer Diskurse sind normative Bedeutungen an der Produktion und Legitimierung einer ganz bestimmten Version sozialer Realität beteiligt und tragen dazu bei, dass bestimmte Praktiken als gut oder normal verstanden werden. Zudem stehen die scheinbar angemessenen Praktiken, die durch Normen konstituiert werden, nicht isoliert da, sondern sind etwa verknüpft mit anderen diskursiven Elementen, etwa bestimmten Vorstellungen des Subjekts, das diese Praktiken ausüben soll/ kann. Normen sind also Bestandteil einer ganz bestimmten Realität, die durch den ihnen zugrunde liegenden Diskurs konstituiert wird, und die konstitutive Wirkung dieses Diskurses kann Bestandteil einer Normenforschung sein. Als Bestandteile hegemonialer Diskurse partizipieren diese Normen aber nicht nur an der produktiven, sondern auch an der repressiven Wirkung dieser Diskurse und delegitimieren andere Praktiken, Subjektpositionen und Objekte als schlecht, unnatürlich, oder überschreiben diese Konstruktionen. Die repressive Seite globaler Normen zu erforschen ist folglich ebenfalls zentraler Bestandteil einer kritischen Normenforschung. In dieser Hinsicht lässt sich bei einer kritischen Beleuchtung globaler Normen etwa fragen, welche Subjektpositionen mit diesen Normen verknüpft sind und naturalisiert werden, wie also die Subjekte konstituiert sind, die die jeweiligen Praktiken ausüben sollen und auch, wie zugleich andere Subjektpositionen überschrieben und marginalisiert werden.

Wie eine solche kritische Untersuchung globaler Normen aussehen kann, wird im Folgenden kurz am Beispiel der globalen Versöhnungsnorm illustriert.

\section{Kritische Normenforschung in der Praxis: Ein Blick auf die globale Versöhnungsnorm}

Die nationale Versöhnung von Postkonfliktgesellschaften anzustreben und zu fördern wird in der internationalen Politik weitgehend als erwünschtes und angemessenes Verhalten betrachtet, man könnte es also als akzeptierte Norm verstehen (vgl. Moon 2008). Die Versöhnungsnorm hat in den vergangenen zwanzig Jahren eine erstaunliche Karriere durchlaufen. Spätestens seit dem südafrikanischen Versöhnungsprozess Mitte der 1990er Jahre geht man davon aus, dass Versöhnung für Postkonfliktgesellschaften wün- 
schenswert ist und dass Versöhnung durch sogenannte Wahrheits- und Versöhnungskommissionen (Truth and Reconciliation Commissions, TRCs) zu erreichen ist, in der die vermeintlichen Opfer und Täter vergangener Menschenrechtsverbrechen öffentlich die Wahrheit über ihre vergangenen Erfahrungen berichten und dadurch von ihren Traumata geheilt und schließlich versöhnt werden (Moon 2008; Humphrey 2002). Zahlreiche Postkonfliktländer haben in den vergangenen Jahren TRCs eingerichtet, darunter Sierra Leone, Liberia, Osttimor, Ghana und Serbien, um nur einige Beispiele zu nennen. Mittlerweile, so Claire Moon, ist das Streben nach nationaler Versöhnung durch Wahrheitskommissionen von einer möglichen zur allgemein erwarteten Reaktion auf Krieg und Unterdrückung geworden (Moon 2008: 2; vgl. auch Christodoulidis/Veitch 2007; Ben-Josef Hirsch 2007), die von diversen trans- und internationalen Akteuren oder NormunternehmerInnen wie dem International Center for Transitional Justice (ICTJ), den Vereinten Nationen oder auch der Europäischen Union als angemessene Antwort auf Gewalt und Unterdrückung propagiert und unterstützt wird (Renner 2013).

Aus Sicht der konstruktivistischen Normenforschung ließe sich hinsichtlich der Versöhnungsnorm nun fragen, wie diese Norm sich global verbreitet hat, welche NormunternehmerInnen am Diffusionsprozess mitgewirkt haben, ob Framing- oder Überzeugungsprozesse ausschlaggebend waren und unter welchen Umständen neue Postkonfliktländer die Versöhnungsnorm in ihren Friedensprozessen adaptieren. Die Geltungsansprüche und politischen Konsequenzen der Versöhnungsnorm lassen sich aus dieser Perspektive aber kaum zum Thema einer wissenschaftlichen Untersuchung machen, denn die Hinterfragung globaler Normen, ihrer Geltungsansprüche und ihrer Konsequenzen wird hier zum großen Teil ausgeblendet. Genau danach fragt aber eine poststrukturalistisch inspirierte Normenforschung.

Aus poststrukturalistischer Perspektive stellt die Versöhnungspraxis Teil eines globalen Versöhnungsdiskurses dar, der im Kontext von Postkonfliktpolitik hegemonial geworden ist und zur Technologisierung der Versöhnungspraxis geführt hat, d.h. zu ihrer Entpolitisierung, so dass diese Praxis relativ unumstritten in immer neue Postkonflikt-Kontexte getragen und dort routinemäßig implementiert wird. Eine kritische Untersuchung dieser Technologie und des Versöhnungsdiskurses zielt dann einerseits darauf ab, die Versöhnungsnorm zu denaturalisieren und zu zeigen, dass der Imperativ zur Versöhnung weder eine gegebene Bedeutung noch einen intrinsischen moralischen Wert besitzt, sondern Bestandteil eines kontingenten Versöhnungsdiskurses ist, dessen Hegemonie und Privilegierung auf der Delegitimierung anderer Konstruktionen beruht. Zweitens ist das Ziel 
einer kritischen Untersuchung, die Versionen sozialer Realität zu enthüllen, die durch den Versöhnungsdiskurs unterdrückt, überschrieben oder delegitimiert wurden. ${ }^{5}$

\subsection{Versöhnung durch Wabrheit als kontingente Bedeutung - Die Artikulation von Versöbnung in Südafrika}

Südafrika kann als der Ort betrachtet werden, an dem die globale Versöhnungspolitik, wie wir sie heute kennen, normativ autorisiert wurde, denn der südafrikanische Versöhnungsprozess, der im Kontext der Transition von der Apartheid zur Demokratie stattfand, gilt heute allgemein als Prototyp und vielversprechendes Vorbild für jegliche Versöhnungspolitik (vgl. etwa Moon 2008; Rotberg/Thompson 2000; Humphrey 2002; Ben-Josef Hirsch 2007). Am Beispiel Südafrikas lässt sich nun rekonstruieren, wie Versöhnung im Laufe der politischen Auseinandersetzungen um die Neuordnung Südafrikas als autoritativer leerer Signifikant artikuliert wurde, der mit anderen Signifikanten wie etwa Heilen, Wabrheit-Sprechen, Opfer und Täter in eine Äquivalenzbeziehung gesetzt und somit inhaltlich gefüllt wurde und wie zugleich Forderungen nach Gerechtigkeit und Bestrafung der Apartheid-FunktionärInnen mit Versöhnung kontrastiert und damit delegitimiert wurden. Zudem zeigt eine solche Analyse, dass die Vorstellung, Versöhnung sei wichtig und durch öffentliches Wahrheit-Sprechen erreichbar, in Südafrika so zunächst nicht existiert hat. Vielmehr war die Idee, Südafrika solle sich versöhnen, zunächst höchst umstritten und die Interpretation sowohl der Autorität als auch der Bedeutung von Versöhnung änderte sich kontinuierlich im Prozess der politischen Auseinandersetzung während der Transition.

Noch in den sehr frühen Tagen der Transition äußerte sich das sogenannte Kairos Dokument, das 1985 von einer Gruppe von Theologen verschiedener Konfessionen veröffentlicht wurde und kritisch zur politischen Situation des Landes Stellung nahm, äußerst skeptisch gegenüber dem Ziel der Versöhnung in Südafrika. In einer Gesellschaft, in der die brutale Unterdrückung der Schwachen durch die Starken an der Tagesordnung ist, sei die Idee einer Versöhnung dieser beiden Gruppen »a total betrayal of all that Christian faith has ever meant« (Kairos 2007). Was Südafrika vor

5 Aus Platzgründen kann die empirische Rekonstruktion in den folgenden Abschnitten dieses Beitrags nur skizzenhaft erfolgen. Für eine ausführliche Darstellung siehe Renner 2013: Kap. 2, 5. 
jeder Versöhnung brauche, so die Kairos-Theologen, sei Gerechtigkeit und die Bestrafung derer, die am unterdrückerischen Regime mitgewirkt haben. Wenn überhaupt, so die Theologen, müsse wahre Versöhnung angestrebt werden und diese sei nur auf der Basis von Gerechtigkeit erreichbar (Kairos 2007; vgl. auch Moon 2008: 32-35).

In diesen Passagen konstruiert das Kairos Dokument Versöhnung auf zweierlei Art und Weise: Einerseits als illegitimes Anderes zu der gewünschten Gerechtigkeit durch Bestrafung und andererseits als legitimes Ziel (wahre Versöhnung), das in einer positiven semantischen Beziehung mit Gerechtigkeit steht und durch diese hervorgerufen wird. Obwohl die Kairos-Theologen mit ihren Forderungen und Artikulationen nicht allein waren, wurden die Rufe nach Gerechtigkeit und Bestrafung im Laufe der Zeit mit der Artikulation des Versöhnungsdiskurses und der Privilegierung von Versöhnung als hegemonialem leeren Signifikanten unterdrückt. Der entstehende Versöhnungsdiskurs propagierte Versöhnung als einzig gutes Ziel für das neue Südafrika und delegitimierte zugleich Forderungen nach Gerechtigkeit und Bestrafung, indem er alle Arten von Bestrafung im Rahmen dieses Diskurses im Sinne von Rache konstruierte (Wilson 2001; Moon 2008).

Während des weiteren Artikulationsprozesses im Laufe der Transition ist zu beobachten, wie Versöhnung Schritt für Schritt zum leeren Signifikanten, zum gemeinsamen Bezugspunkt und zur vagen politischen Vision der politischen Kontrahenten der Transitionsverhandlungen wurde. In den politischen Auseinandersetzungen der National Party (NP) unter Präsident Willem Frederik de Klerk einerseits und dem African National Congress (ANC) unter seinem Vorsitzenden Nelson Mandela andererseits, wurde Versöhnung von beiden Parteien immer mehr als zentraler politischer Schlüsselbegriff bemüht, der das gemeinsame Ziel repräsentieren und die jeweiligen politischen Forderungen der beiden Seiten rechtfertigen sollte. Dabei wurde der Begriff der Versöhnung mit ständig wechselnden Bedeutungen versehen, indem er mit verschiedenen Forderungen in Beziehung gesetzt wurde, die von den beiden Parteien vorgebracht wurden. So wurde Versöhnung alternierend herangezogen, um Forderungen nach politischen Verhandlungen und Kompromiss zwischen den AntagonistInnen Nachdruck zu verleihen, um die Freilassung politischer Gefangener zu rechtfertigen, um nach einem Ende der Apartheid-Gesetzgebung zu rufen, oder um für eine Machtteilung zwischen den Parteien zu plädieren (vgl. Renner 2013). De Klerk verwies etwa in seiner Eröffnungsrede vor dem Parlament 1990 auf die Notwendigkeit von Verhandlungen mit dem/der damaligen politischen GegnerIn und rechtfertigte diese Forderung mit dem Verweis auf Versöhnung: 
"Practically every leader agrees that negotiation is the key to reconciliation, peace and a new and just dispensation [...] Against this background I committed the Government during my inauguration to giving active attention to the most important obstacles in the way of negotiation "(de Klerk 1990).

1992 interpretierte er dann Versöhnung im Sinne der Freilassung politischer Gefangener:

"In a spirit of reconciliation, we will now also release all prisoners falling outside those guidelines [...] who have committed crimes with a political motivation. This will apply to prisoners irrespective of their political affiliation. [...] [the legislation] will deal aquitably with those prisoners whose release can make a contibution [sic!] to reconciliation" (de Klerk 1992)

Beide Zitate illustrieren, wie positive semantische Beziehungen, etwa is the key to oder make a contribution to, eine Äquivalenzbeziehung zwischen Versöhnung und den jeweiligen Forderungen herstellen und Versöhnung zugleich mit Bedeutung versehen. Dennoch unterscheiden sich diese Artikulationen deutlich von dem, was heute allgemein unter einer Versöhnungspolitik verstanden wird.

Fixiert wurde das vage Ideal der Versöhnung erstmals in der Interimsverfassung von 1993, wo es als Ziel einer Amnestieklausel festgeschrieben wurde. Von da an begann ein Prozess der Neuinterpretation von Versöhnung, der vor allem von der südafrikanischen Zivilgesellschaft vorangetrieben wurde, und im Rahmen dessen man zu dem Verständnis kam, das heute im globalen Diskurs vorherrscht: Durch die spezifischen Forderungen zivilgesellschaftlicher AkteurInnen in Bezug darauf, wie das Amnestiegebot der Verfassung umgesetzt werden sollte, wurde Versöhnung nun konstruiert als ein Aufarbeiten der Vergangenheit durch einen öffentlichen Wahrheitsprozess. Dieser sollte ohne Rache, also ohne die Bestrafung der TäterInnen vergangener Gewalt vonstattengehen und vielmehr auf Vergebung durch die Opfer der Gewalt und auf dem Heilen der Traumata von den vermeintlichen Opfern und TäterInnen durch gemeinsames Klagen aufbauen (für eine detaillierte Darstellung vgl. Renner 2013). So wurde zu dieser Zeit etwa argumentiert:

"Forgiveness and reconciliation should thus be understood as the conclusions of a process of reconstructing the moral order that is more healthy than punishment [...]. A society cannot reconcile itself on the grounds of a divided memory. [...] It would thus be important to reveal the truth and so build a moral order [...] without truth and acknowledgement reconciliation is not possible« (Zalaquett 1997: 11-13). 
Die semantischen Beziehungen, die hier zwischen Versöhnung und anderen Signifikanten hergestellt werden, stellen Versöhnung in eine Äquivalenzbeziehung zu forgiveness, reveal the truth und acknowledgement und kontrastieren es zugleich mit punishment. Diese Interpretation von Versöhnung wurde später durch die Sprache und die Praktiken der südafrikanischen TRC reproduziert und gefestigt. Zudem artikulierte die TRC die Subjektpositionen des Opfers und Täters vergangener Menschenrechtsverbrechen als ProtagonistInnen eines solchen Versöhnungsprozesses (vgl. im Detail Renner 2013: Kapitel 2; Moon 2008; Verdoolaege 2008).

Das obige Zitat spiegelt das Verständnis von Versöhnung wider, wie es heute global akzeptiert ist. Zugleich zeigt der Blick auf den Verlauf des Artikulationsprozesses von Versöhnung in Südafrika, dass diese Interpretation zu Beginn nicht existent war. Versöhnung war in der frühen Phase der Transition eine von vielen Forderungen, die hinsichtlich der Zukunft des Landes gestellt wurden, etwa neben Forderungen nach der Bestrafung der Regimemitglieder. Obwohl die Forderung nach Versöhnung nicht unumstritten war, wurde sie im Laufe der Transitionsverhandlungen privilegiert gegenüber den Forderungen nach Bestrafung, die im Sinne von (illegitimer und gefährlicher) Rache interpretiert und dadurch delegitimiert wurden. Zudem wurde unsere heutige Interpretation von Versöhnung im Sinne öffentlichen Wahrheit-Sprechens erst im Laufe der Zeit artikuliert durch die politischen Forderungen, die v.a. von der südafrikanischen Zivilgesellschaft hinsichtlich der Umsetzung der Amnestieklausel geäußert wurden. Unser heutiges Verständnis, Versöhnung sei in Postkonfliktsituationen wünschenswert und durch öffentliche Wahrheitsprozesse erreichbar, kann somit als kontingente Interpretation gesehen werden, statt als existente und scheinbar gute Norm, die heute oft unkritisch in Postkonfliktländer getragen wird.

\subsection{Die Macht des globalen Versöbnungsdiskurses in lokalen Postkonflikt- Kontexten}

Neben der Enthüllung der Kontingenz unserer heutigen Versöhnungsnorm zielt die poststrukturalistische Perspektive auch darauf ab, den politischen Charakter dieser Norm bzw. des ihr zugrunde liegenden Versöhnungsdiskurses zu erforschen und aufzuzeigen, wie dieser Diskurs eine ganz bestimmte Version sozialer Realität produziert und dabei andere Versionen überschreibt und marginalisiert. Der globale Versöhnungsdiskurs wird primär in Postkonfliktgesellschaften implementiert und es lässt sich daher besonders gut in solchen lokalen Kontexten untersuchen, wie dieser 
Diskurs seine Macht entfaltet, indem er eine spezielle Versöhnungsrealität implementiert und dabei andere Interpretationen der Postkonfliktrealität unterdrückt. Dies werde ich im Folgenden knapp am Beispiel Sierra Leones illustrieren, wo der globale Versöhnungsdiskurs im Laufe der Verhandlungen des Lomé Friedensabkommens 1999 an Einfluss gewann.

Der globale Versöhnungsdiskurs wird primär durch globale AkteurInnen in lokale Postkonfliktkontexte gebracht, die ich an anderer Stelle als globale Versöbnungskoalition bezeichnet habe. Internationale Nichtregierungsorganisationen (NGOs) wie das International Center for Transitional Justice, das UN Hohe Kommissariat für Menschenrechte (OHCHR) und individuelle Mitglieder ehemaliger TRCs, primär aus Südafrika, fungieren als vermeintliche Versöhnungsexperten, bringen das Wissen über Versöhnung in Postkonfliktgesellschaften und helfen beim Aufbau und der Durchführung von TRCs (vgl. im Detail Renner 2013: Kap. 4, 5). Während diese globalen AkteurInnen durch ihren ExpertInnenstatus Definitions- und Handlungsmacht genießen und den Versöhnungsprozess gemäß globalen best practice-Standards gestalten, stellt der Versöhnungsdiskurs auch für die lokale Bevölkerung bestimmte Subjektpositionen bereit.

Wie oben bereits angedeutet, ist die Versöhnungspraxis des öffentlichen Wahrheit-Sprechens im Diskurs mit zwei bestimmten Subjektpositionen verknüpft, nämlich dem Opfer und dem Täter oder der Täterin vergangener Menschenrechtsverbrechen. Der Versöhnungsdiskurs konstituiert Versöhnung als einen Prozess, in dem die Opfer und TäterInnen vergangener Menschenrechtsverbrechen die Wahrheit über ihre Erfahrungen erzählen, ihre Traumata heilen und sich versöhnen (sollen) (vgl. Moon 2008; Renner 2013). Opfer und TäterIn sind also die Subjektpositionen, die den Menschen im Kontext des Versöhnungsprozesses zur Identifikation zur Verfügung gestellt werden.

Im Fall Sierra Leones lässt sich nun beobachten, wie die produktive Macht des Versöhnungsdiskurses die Individuen der Postkonfliktgesellschaft dort als Opfer und TäterIn vergangener Menschenrechtsverbrechen konstituierte, die vor allem ein Bedürfnis nach Versöhnung und therapeutischer Heilung haben. Die Identifikation der Menschen mit diesen Subjektpositionen und ihre Anerkennung als solche, geschah primär durch die Prozesse und Praktiken der Sierra Leonischen TRC (vgl. ausführlich Renner 2013: Kap. 5), als also die globale Norm Versöhnung durch öffentliche Wahrheitsprozesse in Sierra Leone implementiert wurde. Zwei Interpellationsmechanismen der TRC sind besonders auffällig: Einerseits verlangte die Teilnahme am öffentlichen Aufarbeitungsprozess von den Menschen, sich als Opfer oder TäterIn zu klassifizieren und drängte sie dadurch in diese Subjektpositionen. Um etwa die Anmeldeformulare der TRC auszu- 
füllen, in denen es spezielle Sektionen für Opfer und Sektionen für TäterInnen gab, mussten die Menschen sich entscheiden, als was sie sich selbst sahen: als Opfer, TäterIn, oder eine Kombination aus beidem (SLTRC 2004b: Volume 1, 194-230). Die Interpellation der Menschen mit den neuen Subjektpositionen des Versöhnungsdiskurses erfolgte andererseits auch in den öffentlichen Anhörungen, wo die Kommissare der TRC die ZeugInnen immer wieder als Opfer und TäterInnen ansprachen und ihnen diese Identitäten explizit zuwiesen. So sagte etwa der Vorsitzende der TRC, Bischof Humper, in einer Anhörung zu einem Zeugen:

"You and your people became victims. And you endured and endured and it became non-endurable any longer! So you committed yourself to what ultimately came to be called CDF. So you became a member of CDF. Is that right? And CDF are perpetrators, so you are a perpetrator as well. You are a victim-perpetrator!« (Humper quoted in Kelsall 2005: 375).

Die konstruktive Seite der diskursiven Macht, die die lokale Bevölkerung als Opfer und TäterInnen vergangener Menschenrechtsverbrechen konstituierte, hatte allerdings auch insofern einen repressiven Aspekt, als die neuen Subjektpositionen zugleich andere Subjektpositionen überschrieb, die in der Postkonfliktgesellschaft Sierra Leones bis dahin vorgeherrscht hatten (vgl. ausführlich Renner 2013: Kap. 5). In ihren eigenen Erzählungen bei den Anhörungen und Befragungen der TRC beschrieben die Menschen sich und ihre MitbürgerInnen zunächst nicht als Opfer und TäterInnen vergangener Menschenrechtsverbrechen, sondern vielmehr als Subjekte des Bürgerkrieges. Die Erzählungen der Vergangenheit handelten zunächst von RebellInnen, SoldatInnen, ZivilistInnen oder KollaborateurInnen, die auf der einen oder anderen Seite am Krieg beteiligt waren oder versuchten sich aus diesem herauszuhalten und von deren politischen Positionen. Die folgende Passage aus einer Aussage vor der TRC, in der der Zeuge über die zweite Phase des Krieges berichtet, illustriert diese Subjektpositionen gut:

"In this phase, the civilians were targets of both the rebels and soldiers. Rebels claimed that the civilians were disclosing their whereabouts to soldiers and soldiers accused the civilians of being rebel collaborators" (SLTRC 2004a: 175-176).

Dieses Zitat illustriert, dass Opfer und TäterInnen in den Aussagen vor der TRC zunächst keine Rolle spielten. Stattdessen war es der Versöhnungsdiskurs selbst, der durch die Praktiken der TRC eine Neuinterpretation der Vergangenheit und Gegenwart ermöglichte und die Menschen als Opfer und/oder TäterInnen konstruierte. 
Die Reinterpretation der Sierra Leonischen Bevölkerung als Opfer und/ oder TäterInnen vergangener Menschenrechtsverbrechen und die gleichzeitige Neutralisierung der Identitäten als RebellIn, SoldatIn oder ziviler KollaborateurIn zog noch weitere Konsequenzen mit sich, da diese früheren Identitäten aus dem Bürgerkriegsdiskurs mit bestimmten politischen Haltungen und Forderungen verknüpft waren. So hatte etwa die Revolutionary United Front (RUF) - nach eigener Aussage - gegen die Regierung rebelliert, da Forderungen der Bevölkerung, etwa nach Good Governance, ökonomischer Gerechtigkeit, oder auch politischer Macht und Partizipation über lange Zeit ungehört geblieben waren. Eben diese Forderungen hatten der RUF zunächst auch Unterstützung und Rückhalt in der Bevölkerung verschafft. Insgesamt wird der Bürgerkrieg Sierra Leones in der Literatur häufig interpretiert als Folge langjähriger Korruption, Misswirtschaft und Benachteiligung sowie politischer Exklusion vor allem der ländlichen Bevölkerung (Conteh-Morgan/Dixon-Fyle 1999: 127-128; Pham 2006: 86). Indem der Versöhnungsdiskurs, der durch die Versöhnungspraktik und die Einrichtung der TRC ins Land gebracht wurde, nun die politischen Identitäten der Sierra Leonischen Bevölkerung überschrieb und sie mit den Subjektpositionen des Opfers und des/der TäterIn vergangener Menschenrechtsverbrechen ersetzte, neutralisierte er auch die politischen Forderungen, die von den Subjekten des Bürgerkrieges erhoben wurden, und ersetzte sie durch das eher apolitische Bedürfnis von Opfern und TäterInnen nach Versöhnung und symbolischer Heilung.

Insgesamt, so lässt sich aus poststrukturalistischer Perspektive argumentieren, wirkte der Versöhnungsdiskurs also de-politisierend für die Postkonfliktgesellschaft Sierra Leones. Er konstruierte die Menschen als Opfer und TäterInnen vergangener Menschenrechtsverbrechen, die vor allen Dingen Versöhnung und therapeutische Heilung benötigen. Die politischen und ökonomischen Probleme und Forderungen, die von der Bevölkerung zuvor erhoben wurden und zum Teil auch Auslöser des Bürgerkrieges waren, werden dabei jedoch diskursiv überschrieben und dann politisch zugunsten symbolischer Restitutionsleistungen marginalisiert.

Letztendlich produziert der Versöhnungsdiskurs, der sich v.a. in der Versöhnungsnorm materialisiert, durch seine produktive Macht die Bedingungen seiner eigenen Notwendigkeit: Er geht davon aus, dass Bürgerkriege und Konflikte Opfer und TäterInnen vergangener Menschenrechtsverletzungen hinterlassen, die vor allem ein Bedürfnis nach Heilung, Trost und Versöhnung haben und somit einen Versöhnungsprozess notwendig machen. Zugleich produziert der Versöhnungsprozess diese Subjekte und ihre Bedürfnisse aber selbst und bietet die benötigten Leistungen, nämlich Heilung und Versöhnung gleich symbolisch an, indem er TRCs mit öf- 
fentlichen Wahrheitsprozessen, gemeinsamem Klagen und öffentlichem Trost inszeniert. Der Versöhnungsdiskurs macht Postkonfliktsituationen somit in gewisser Weise regierbar: Er definiert die Bedürfnisse und Probleme vor Ort im Sinne eines Mangels an Versöhnung und Heilung und liefert zugleich die Technologien, die diesen Mangel zu beheben scheinen. Zugleich überschreibt er politische Identitäten und soziale Forderungen und Probleme, die möglicherweise zum Ausbruch des Bürgerkriegs beigetragen haben, nun aber nicht politisch adressiert werden.

\section{Schlussbemerkung}

Die poststrukturalistische Perspektive auf das, was zuweilen als globale Versöhnungsnorm verstanden wird, sollte illustrieren, wie eine poststrukturalistisch inspirierte Untersuchung normativer Bedeutungen aussehen kann. Die poststrukturalistische Diskurstheorie ist sicherlich nicht der einzige Weg, den man für eine kritische Form der Normenforschung beschreiten kann. Dennoch versprechen die poststrukturalistische Perspektive und Lesart internationaler Normen andere Einsichten und Ergebnisse hinsichtlich deren Herausbildung und globaler Verbreitung.

Die poststrukturalistische Perspektive, die in diesem Beitrag vorgeschlagen wurde, führt zu einer anderen und kritischeren Bewertung der globalen Versöhnungsnorm als die oben skizzierten sozialkonstruktivistischen Ansätze, die nach den Prozessen und Bedingungen der Diffusion dieser Norm fragen und diese anhand von akteurszentrierten Modellen zu erklären versuchen. Indem sie ihr Augenmerk nicht nur auf die Norm der Versöhnung, d.h. den Verhaltensstandard des Wahrheit-Sprechens und TRCSchaffens selbst legt, sondern versucht, den weiteren Diskurs zu erfassen, in den dieser Standard eingebettet ist, ermöglicht sie eine umfassendere Analyse und lenkt den Blick auch auf die Subjektpositionen von Opfer und TäterIn, die mit der Versöhnungsnorm verknüpft sind. Indem sie die produktive und repressive Macht des Versöhnungsdiskurses in den Blick nimmt, beleuchtet sie Konsequenzen, die einer traditionelleren sozialkonstruktivistischen Perspektive entgehen.

Insgesamt zieht die poststrukturalistische Perspektive die Annahme in Zweifel, die globale Versöhnungsnorm und die zugehörige angemessene Praxis, Versöhnung in Postkonfliktgesellschaften durch Wahrheitskommissionen und das Heilen von Opfern und TäterInnen zu fördern, sei eine notwendigerweise gute oder auch nur eine neutrale und unschuldige Praktik. Stattdessen, so lässt sich aus poststrukturalistischer Perspektive argumentieren, ist die Versöhnungsnorm eingebettet in den Versöhnungsdis- 
kurs und ist somit KomplizIn seiner produktiven und repressiven Macht. Damit ist die Versöhnungsnorm als hoch politisch zu betrachten, da sie Konsequenzen zeitigt, die in der Politik und auch in der wissenschaftlichen Forschung oft übersehen werden, da Versöhnung durch Wahrheit meist unhinterfragt als gute, angemessene Friedenspraxis akzeptiert wird.

\section{Literatur}

Bailey, Jennifer L. 2008: Arrested Development: The Fight to End Commercial Whaling as a Case of Failed Norm Change, in: European Journal of International Relations 14: 2, 289-318.

Ben-Josef Hirsch, Michal 2007: Agents of Truth and Justice. Truth Commissions and the Transitional Justice Epistemic Community, in: Chandler, David/Heins, Volker (Hrsg.): Rethinking Ethical Foreign Policy: Pitfalls, Possibilities and Paradixes, London, 184-205.

Christodoulidis, Emilios A./Veitch, Scott 2007: Introduction, in: Veitch, Scott (Hrsg.): Law and the Politics of Reconciliation, Aldershot, 1-8.

Conteh-Morgan, Earl/Dixon-Fyle, Mac 1999: Sierra Leone at the End of the Twentieth Century. History, Politics, and Society, New York.

Cox, Robert W. 1981: Social Forces, States and World Orders: Beyond International Relations Theory, in: Millennium: Journal of International Studies 10: 2, 126-155.

de Klerk, Frederik Willem 1990: Address by the State President, Mr. FW de Klerk, DMS, at the Opening of the Second Session of the Ninth Parliament of the Republic of South Africa, Cape Town, 2 February 1990, in: http://www.artsrn.ualb erta.ca/amcdouga/Hist247/winter_2011/document\%20analysis/de_klerk_address _1990.pdf, 2. September 2014.

de Klerk, Frederik Willem 1992: Letter from State President FW de Klerk to Nelson Mandela President of the ANC, 24 September 1992, in: http://www.anc.org.za/a ncdocs/history/transition/fwletter092492.html, previously available at this website.

Deitelhoff, Nicole 2006: Überzeugung in der Politik. Grundzüge einer Diskurstheorie internationalen Regierens, Frankfurt am Main.

Deitelhoff, Nicole 2009: The Discursive Process of Legalization: Charting Islands of Persuasion in the ICC Case, in: International Organization 63: 1, 33-65.

Edkins, Jenny 1999: Poststructuralism \& international Relations. Bringing the Political Back in, London.

Engelkamp, Stephan/Glaab, Katharina/Renner, Judith 2012: 'In der Sprechstunde'. Wie (kritische) Normenforschung ihre Stimme wiederfinden kann, in: Zeitschrift für Internationale Beziehungen 19: 2, 101-129.

Epstein, Charlotte 2008: The Power of Words in International Relations. Birth of an Anti-Whaling Discourse, Cambridge, Massachussetts. 
Fairclough, Norman 2003: Analysing Discourse. Textual Analysis for Social Research, London \& New York.

Finnemore, Martha/Sikkink, Kathryn 1998: International Norm Dynamics and Political Change, in: International Organization 52: 4, 887-917.

Florini, Ann 1996: The Evolution of International Norms, in: International Studies Quarterly 40: 3, 363-389.

Guzzini, Stefano 2000: A Reconstruction of Constructivism in International Relations, in: European Journal of International Relations 6: 2, 147-182.

Guzzini, Stefano 2005: The Concept of Power: A Constructivist Analysis, in: Millennium: Journal of International Studies 33: 3, 495-521.

Hanrieder, Tine 2008: Moralische Argumente in den Internationalen Beziehungen. Grenzen einer verständigungstheoretischen 'Erklärung' moralischer Debatten, in: Zeitschrift für Internationale Beziehungen 15: 2, 161-186.

Howarth, David 2004: Hegemony, Political Subjectivity, and Radical Democracy, in: Critchley, Simon/Marchart, Oliver (Hrsg.): Laclau. A Critical Reader, London and New York, 256-276.

Howarth, David/Stavrakakis, Yannis 2000: Introducing Discourse Theory and Political Analysis, in: Howarth, David/Norval, Aletta J./Stavrakakis, Yannis (Hrsg.): Discourse Theory and Political Analysis. Identities, Hegemonies and Social Change, Manchester, 1-23.

Humphrey, Michael 2002: The Politics of Atrocity and Reconciliation. From Terror to Trauma, London \& New York.

Kairos 2007: The Kairos Document, in: Doxtader, Erik/Salazar, Philippe-Joseph (Hrsg.): Truth and Reconciliation in South Africa. The Fundamental Documents, Claremont, 50-56.

Keck, Margaret W./Sikkink, Kathryn 1998: Activists Beyond Borders. Advocacy Networks in International Politics, Ithaca and London.

Kelsall, Tim 2005: Truth, Lies, Ritual: Preliminary Reflections on the Truth and Reconciliation Commission in Sierra Leone, in: Human Rights Quarterly 27: 2, 361-391.

Klotz, Audie 1995: Norms in International Relations. The Struggle Against Apartheid, Ithaca \& London.

Laclau, Ernesto 2004: Glimpsing The Future, in: Critchley, Simon/Marchart, Oliver (Hrsg.): Laclau. A Critical Reader, London and New York, 279-328.

Laclau, Ernesto 2007: Emancipation(s), London.

Laclau, Ernesto/Mouffe, Chantal 2001: Hegemony and Socialist Strategy. Towards a Radical Democratic Politics, London.

Liberia 2003: Comprehensive Peace Agreement Between the Government of Liberia and the Liberians United for Reconcilation and Democracy (LURD) and the Movement for Democracy in Liberia (MODEL) and Political Parties, in: http:/www.usip.org/files/file/resources/collections/peace_agreements/liberia_08 182003.pdf, 2 September 2014. 
Milliken, Jennifer 1999: The Study of Discourse in International Relations: A Critique of Research and Methods, in: European Journal of International Relations 5: 2, 225-254.

Moon, Claire 2008: Narrating Political Reconciliation. South Africa's Truth and Reconciliation Commission, Plymouth, UK.

Park, Susan 2005: Norm Diffusion within International Organizations: a Case Study of the World Bank, in: Journal of International Relations and Development 8: 2, 111-141.

Payne, Rodger A. 2001: Persuasion, Frames and Norm Construction, in: European Journal of International Relations 7: 1, 37-61.

Pham, J. Peter 2006: The Sierra Leonean Tragedy. History and Global Dimensions, New York.

Price, Richard 1995: A Genealogy of the Chemical Weapons Taboo, in: International Organization 49: 1, 73-103.

Renner, Judith 2013: Discourse, Normative Change, and the Quest for Reconciliation in Global Politics, Manchester.

Risse, Thomas 2000: 'Let's Argue!': Communicative Action in World Politics, in: International Organization 54: 1, 1-39.

Risse, Thomas/Ropp, Stephen C./Sikkink, Kathryn (Hrsg.) 1999: The Power of Human Rights. International Norms and Domestic Change, Cambridge, MA.

Risse, Thomas/Sikkink, Kathryn 1999: The Socialization of International Human Rights Norms into Domestic Practices: Introduction in: Risse, Thomas/Ropp, Stephen C./Sikkink, Kathryn (Hrsg.): The Power of Human Rights. International Norms and Domestic Change, Cambridge, 1-38.

Rotberg, Robert I./Thompson, Dennis (Hrsg.) 2000: Truth v. Justice. The Morality of Truth Commissions, Princeton \& Oxford.

Sandholtz, Wayne 2008: Dynamics of International Norm Change: Rules against Wartime Plunder, in: European Journal of International Relations 14: 1, 101-131.

SLTRC 2004a: SLTRC Report: Appendix 3 - Part Three - Transcripts of Thematic and Institutional Hearings, in: http://www.sierra-leone.org/Other-Conflict/APP ENDICES/Appendix\%203\%20Transcript\%20of\%20Public\%20Hearings.pdf, 2 September 2014.

SLTRC 2004b: Witness to Truth: Report of the Sierra Leone Truth and Reconciliation Commission, in: http://www.sierra-leone.org/Other-Conflict/TRCVolume1. pdf, 2. September 2014.

Torfing, Jacob 2005: Discourse Theory: Achievements, Arguments, and Challenges, in: Howarth, David/Torfing, Jacob (Hrsg.): Discourse Theory in European Politics. Identity, Policy and Governance, Hampshire, 1-33.

Verdoolaege, Annelies 2008: Reconciliation Discourse, Amsterdam/Philadelphia.

Wiener, Antje 2004: Contested Compliance: Interventions on the Normative Structure of World Politics, in: European Journal of International Relations 10: 2, 189-234. 
Wiener, Antje 2007a: Contested Meanings of Norms: A Research Framework, in: Comparative European Politics 5, 1-17.

Wiener, Antje 2007b: The Dual Quality of Norms and Governance beyond the State: Sociological and Normative Approaches to 'Interaction', in: Critical Review of International Social and Political Philosophy 10: 1, 47-69.

Wilson, Richard A. 2001: The Politics of Truth and Reconciliation in South Africa, Cambridge.

Zalaquett, José 1997: Why Deal with the Past?, in: Boraine, Alex/Levy, Janet/Scheffer, Ronel (Hrsg.): Dealing with the Past. Truth and Reconciliation in South Africa, Cape Town, 9-15. 
